\title{
MULTIPHYTOADAPTOGENE IMMUNOMODIFYING EFFECT OF LEUKOCYTE INTEGRINS EXPRESSION REGULATION IN CBA MICE WITH SPONTANEOUS HEPATOMAS
}

\author{
(C) Bocharova O.A., Karpova R. V., Bocharov E. V., \\ Ilyenko V. A., Kazeev I. V., Utkina M. V.
}

N. N. Blokhin Cancer Research Center of RAMS, Moscow, Russia

Adhesive interactions between immune and cancer cells are significant for reducing the immune escape mechanism for tumors. The aim of this study was to investigate the leukocyte integrins LFA-1 (CD11a) and Mac-1 (CD11b) expressions on blood lymphocytes in males of CBA inbred mice susceptible to spontaneous hepatomas as well as their regulation by multiphytoadaptogene phytomix-40 (phm-40) under different schemes of administration. Phm-40 consists of components from forty medical herbs extracts. Phm-40 develops wide spectrum of activities including antitumour and immunomodifying effects. The mice of the 1-st group $(n=90)$ were control animals. The mice of the 2 -nd group $(n=40)$ were given $10 \%$ phm-40 solution in drinking water during the first month of life including the final time period of liver differentiation (as preventive application). The mice of the 3 -d group $(n=40)$ were given $10 \%$ phm-40 solution in drinking water starting at the 6-th month of mouse age (when the first carcinomas are generated) by 3 weeks courses with 1 week intervals (as therapeu- tic application). Immune cells were analyzed by quantifying the expression of CD11a and CD11b antigens using flow cytometric study. There were no significant differences between parameters evaluated in all groups at the ages of 4 and 8 months. In the control group of animals LFA-1 and Mac-1 expressions were decreased down to $35,4 \pm 1,6 \%$ and $7,8 \pm 1,0 \%$ correspondingly $\left(p_{8-22}<0,01\right)$ at the age of 22 months. Parameters alterations observed can suppress adhesive interactions between immune effectors and tumour cells. At the same time in the 2-nd and 3-d groups (preventive and therapeutic phm-40 administrations) LFA-1 (40,7 $\pm 1,9 \%$ and $42,3 \pm 2,9 \%$ correspondingly) and Mac-1 (11,5 $\pm 1,1 \%$ and $12,8 \pm 1,7 \%$ correspondingly) expressions were higher than parameters in control group $(p=0,05)$ at the age of 22 months. The results suggest the upregulation of the blood lymphocytes level with leukocyte integrins is due to the elevation of adhesive ligands (ICAM-1, 2 ) in tumours for immune effectors which can kill cancer cells enhancing the organism antitumor inspection.

\section{MULTIPHYTOADAPTOGENE PREPARATION REDUCED IL-6 AND IL-10 SERUM LEVELS IN CBA MICE WITH SPONTANEOUS HEPATOMAS}

\section{(C) Bocharova O. A., Karpova R. V., Bocharov E. V., Ilyenko V. A., Kazeev I. V.}

N. N. Blokhin Cancer Research Center of RAMS, Moscow, Russia

Cytokines are pleiotropic in their biological activities including the immune response. In recent years it is obvious that cytokines mediate immune and tumour cells interactions. Serum cytokines IL-6 and IL-10 as well as their regulation by multiphytoadaptogene preparation phytomix-40 (phm-40) under different schemes of administration were investigated in males of CBA inbred mice with high frequency of spontaneous hepatocarcinomas. Phm-40 consists of components from forty medical herbs extracts. Phm-40 develops wide spectrum of activities including antioxidant, antimutagenic, antitumour and immunomodifying effects. The mice of the 1 -st group $(n=90)$ were control animals. The mice of the 2-nd group $(n=40)$ were given $10 \%$ phm-40 solution in drinking water during the first month of life including the final time period of liver differentiation (as preventive application). The mice of the 3-d group $(n=40)$ were given $10 \%$ phm-40 solution in drinking water starting at the 6-th month of mouse age (when the first carcinomas are generated) by 3 weeks courses with 1 week intervals (as therapeutic application). Serum cytokine concentrations were determined by enzyme-linked immunosorbent assay. There were no differences between parameters evaluated in animals of all groups at the age of 4 and 8 months. The IL- 6 and IL- 10 serum concentrations in control animals were elevated significantly (to $139,1 \pm 6,6 \mathrm{pg} / \mathrm{ml}$ and $60,9 \pm 3,9 \mathrm{pg} / \mathrm{ml}$ correspondingly, $p<0,01)$ at the age of 22 months. However IL- 6 and IL-10 serum levels in the 2 -nd and the 3 -d groups were lower at the age of 22 months than in young animals $(p<0,05)$. Thus early short-term preventive as well as prolonged therapeutic phm-40 administrations lead to reducing IL-6 and IL-10 serum levels. Decrease 
of IL-10 (as immunosupressive cytokine) and IL-6 (which takes part in myoproteosis degradation) serum levels can restore immune cells activity. It was correlated with increasing the leukocyte integrins expression. Down regulation of serum IL-6 and IL-10 cytok- ines in males of CBA inbred mice with high frequency of spontaneous hepatocarcinomas is considered to reduce antitumour antibodies production (which cover tumor cells antigens and diminish immune cells activity) as well as cachexia symptoms.

\title{
NUTRACEUTICALS FROM BEETROOT POMACE EXTRACT WITH ANTIOXIDANT AND HEPATOPROTECTIVE EFFECTS
}

\section{(드anadanović-Brunet Jasna ${ }^{1}$, Ćebović Tatjana², Vulić Jelena ${ }^{1}$, Ćetković Gordana ${ }^{1}$, Djilas Sonja $^{1}$}

${ }^{1}$ Faculty of Technology, University of Novi Sad, Bulevar cara Lazara 1, 21000 Novi Sad, Serbia

${ }^{2}$ Faculty of Medicine, Clinical centre of Vojvodina, Hajduk Veljkova 3, Novi Sad, Serbia

The beetroot pomace (BPE), residue from the juice processing, is promising source of bioactive compounds with antioxidant, hepatoprotective and antitumor properties. The aim of this research was to investigate the antioxidant activity of BPE and its effect on biochemical parameters of oxidative stress evaluated in liver homogenate of rats. The antioxidant activity of BPE, including reducing power and DPPH free radical scavenging activity were determined spectrophotometrically. The effects of BPE on several biochemical parameters of oxidative stress were evaluated in liver homogenate of rats treated with $\mathrm{CCl}_{4}$ as a free radical generator: LPx intensity, content of GSH and activities of GSH-Px, GR, Px, XOD and CAT. BPE was able to scavenge DPPH radicals $\left(E_{50}{ }^{D P P H}=3.4 \mu \mathrm{g} / \mathrm{ml}\right)$. The reducing power of BPE increased with increasing the concentration. The BPE, combined with $\mathrm{a} \mathrm{Cl}_{4}$, expressed a clear and dose-dependent suppressive effect against lipid peroxidation in liver homogenate. Simultaneous application of extract and $\mathrm{CCl}_{4}$ has led to a significant decrease in GSH-Px levels, keeping the GSH content in liver homogenate close to physiological level. Similar impact on GR activity has been observed in liver of the animals. The catalase activity was decreased after treatment with $\mathrm{BPE}$ and $\mathrm{CCl}_{4}$ Similar influence on hepatic Px activity has been also observed. Although $\mathrm{CCl}_{4}$ does not alter the activity of hepatic XOD certain increase of XOD activity has been measured after addition of BPE. Obtained results demonstrated that the beetroot pomace extract might possess a specific antioxidant potential, suggesting that it might be used not only as a hepatoprotective agent, but as an active agent in treatment of other disorders caused by oxidative stress.

\section{VALORISATION OF ANTIOXIDANT AND CELL GROWTH ACTIVITIES OF TOMATO WASTE LIPOPHILIC EXTRACTS}

\section{(C) Ćetković Gordana ${ }^{1}$, Savatović Sladjana1, Čanadanović-Brunet Jasna1, ${\text { Djilas Sonja }{ }^{1}, \text { Mandić Anamarija }^{2} \text {, Četojević-Simin Dragana }}^{3}$}

\author{
${ }^{1}$ Faculty of Technology, University of Novi Sad, Bulevar Cara Lazara 1, 21000 Novi Sad, Serbia \\ ${ }^{2}$ Institute for Food Technology in Novi Sad, University of Novi Sad, Bulevar Cara Lazara 1, 21000 Novi Sad, Serbia \\ ${ }^{3}$ Oncology Institute of Vojvodina, Institutski put 4, 21204 Sremska Kamenica, Serbia
}

Tomato, fresh and its products, possess a high nutritional value, due to content of different types of antioxidants: carotenoids, polyphenolics, vitamin $\mathrm{C}$ and vitamin E. According to the fact that different valuable compounds remained in the waste from tomato juice processing, the aim of this research was to investigate the antioxidant and cell growth activities of tomato waste lipophilic extracts (obtained from genotypes - Bačka and Saint Pierre). The lycopene and $\beta$-carotene in tomato waste extracts were identified and quantified by HPLC method by matching their retention times (RT) and on-line ultraviolet (UV) spectra with those of stan- dards. The antioxidant activity of extracts were investigated by measuring 2,2-diphenyl-1-picrylhydrazyl (DPPH) radical scavenging activity by spectrophotometric method. The cell growth activity was measured using the sulforhodamine B colorimetric assay on human cancer HeLa (cervix epitheloid carcinoma) and MCF7 (breast adenocarcinoma) cell lines. The Baka waste extract contained higher st content of lycopene $(13.6 \pm 0.1 \mathrm{mg} / \mathrm{g})$ and $\beta$-carotene $(12.0 \pm 0.3 \mathrm{mg} / \mathrm{g})$. The extracts were able to scavenge DPPH radical and higher antioxidant activity, expressed as $\mathrm{IC}_{50}{ }^{\mathrm{DPPH}}$ value, was obtained in the case of the Bačka waste extract 